\title{
J. WESOŁOWSKI（Warszawa)
}

\section{ARE CONTINUOUS MAPPINGS PRESERVING NORMALITY NECESSARILY LINEAR?}

Abstract. An example of a normal nonlinear continuous function of a normal random variable is given. Also the Cauchy case is considered.

1. Introduction. It is tempting to think that if a Gaussian input is transformed into a Gaussian output then the transformation has to be linear.

Let $X$ be a standard normal $(\mathcal{N}(0,1))$ random variable (rv). Obviously for a function $f: \mathbb{R} \rightarrow \mathbb{R}$ defined by $f(x)=x, x \in \mathbb{R}$, or $f(x)=-x$, $x \in \mathbb{R}$, the transformed $\mathrm{rv} f(X)$ is again standard normal $(f(X) \stackrel{d}{=} \mathcal{N}(0,1))$. Both the functions are linear. However, it is not difficult to find nonlinear functions with the same property. For example, one can consider

$$
f(x)= \begin{cases}-x, & |x|<1 \\ x, & |x| \geq 1\end{cases}
$$

which works also well for any distribution symmetric about zero. A nontrivial bivariate example was given in Shepp (1964). The common features of these nonlinear transforms preserving normality are discontinuities.

Similar problems were studied for the Cauchy distribution in Pitman and Williams (1967), Williams (1969), Arnold (1979) and Neuts (1979) (answering a problem raised in Kotlarski (1977)). The nonlinear functions preserving the Cauchy distribution, given there, were not continuous again. A nice example among them is $f(x)=\tan (n \arctan (x)), n=2,3, \ldots$ For others see also Johnson, Kotz and Balakrishnan (1994).

Since all the known nonlinear mappings preserving normality (or the Cauchy distribution) have discontinuities it is natural to conjecture that under continuity assumption the only possible transforms are linear. The aim of this note is to show that this is not the case again.

1991 Mathematics Subject Classification: Primary 62E05.

Key words and phrases: normal distribution, Cauchy distribution, distributions of functions of random variables. 
2. Distribution preserving transforms. Assume that $X \stackrel{d}{=} f(X) \stackrel{d}{=}$ $\mathcal{N}(0,1)$, where $f: \mathbb{R} \rightarrow \mathbb{R}$ is a continuous function. Is $f$ necessarily linear? It is shown below that the answer is negative (also for the Cauchy distribution).

For any $x \in[-1,1]$ define

$$
b(x)=\Phi\left(\frac{x-3}{4}\right)+\Phi\left(\frac{x+3}{4}\right)-\Phi(x),
$$

where $\Phi$ is the df of the $\mathcal{N}(0,1)$ distribution.

LEMmA. The function $b:[-1,1] \rightarrow[\Phi(-0.5), \Phi(0.5)]$ is strictly decreasing. Hence its inverse function $b^{-1}$ exists.

Proof. Observe that $b$ is differentiable in $(-1,1)$ (continuous in $[-1,1]$ ) and for any $x \in(-1,1)$,

$$
b^{\prime}(x)=\frac{1}{4}\left[\phi\left(\frac{x-3}{4}\right)+\phi\left(\frac{x+3}{4}\right)\right]-\phi(x),
$$

where $\phi$ is the standard normal density. Since for any $x \in(-1,1)$,

$$
\phi\left(\frac{x-3}{4}\right)+\phi\left(\frac{x+3}{4}\right)<2 \phi(0)<4 \phi(1) \leq 4 \phi(x),
$$

it follows that $b^{\prime}<0$ in $(-1,1)$. Hence $b$ is strictly decreasing. Observe that $b(-1)=\Phi(0.5)$ and $b(1)=\Phi(-0.5)$.

Define now a new function $f$ :

$$
f(x)= \begin{cases}x, & |x| \geq 1 \\ 4 x+3, & -1<x<-0.5 \\ B(x), & -0.5 \leq x \leq 0.5 \\ 4 x-3, & 0.5<x<1\end{cases}
$$

where $B=b^{-1} \circ \Phi$ (see Fig. 1).

Proposition. The function $f: \mathbb{R} \rightarrow \mathbb{R}$ is continuous. If $X \stackrel{d}{=} \mathcal{N}(0,1)$ then $f(X) \stackrel{d}{=} \mathcal{N}(0,1)$.

Proof. Continuity is obvious. Also, it is immediate that $P(f(X) \leq$ $x)=\Phi(x)$ for $|x| \geq 1$.

Consider now $|x|<1$. Then $\Phi \circ B^{-1}(x)=b(x)$ and consequently

$$
\begin{aligned}
P(f(X) \leq x) & =P\left(X \leq \frac{x-3}{4}\right)+P\left(B^{-1}(x) \leq X \leq \frac{x+3}{4}\right) \\
& =\Phi\left(\frac{x-3}{4}\right)+\Phi\left(\frac{x+3}{4}\right)-\Phi\left(B^{-1}(x)\right) \\
& =b(x)+\Phi(x)-b(x) .
\end{aligned}
$$




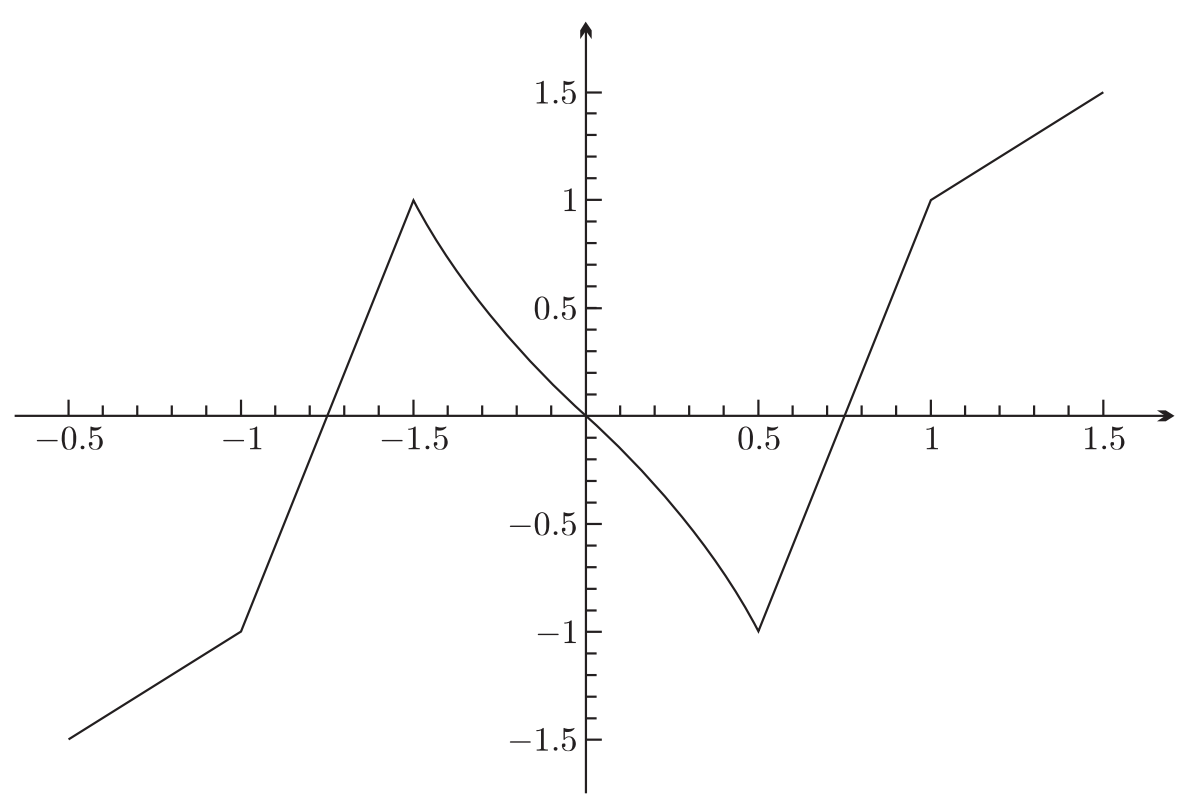

Fig. 1. The graph of $f$

Remarks. 1. Assume that $\Phi(x)=0.5+\pi^{-1} \arctan (x)$ and $\phi(x)=$ $\left(\pi\left(1+x^{2}\right)\right)^{-1}, x \in \mathbb{R}$, i.e. $\Phi$ and $\phi$ are df and pdf of the standard Cauchy distribution, respectively. Then the formula (2) gives an example of a Cauchy preserving nonlinear continuous function since again $b^{\prime}<0$ via the inequalities in $(1)$ with $\phi(0) \leq 2 \phi(1)$ (instead of the strict inequality).

2. A similar construction is valid for any symmetric distribution with a df $\Phi$ and a unimodal density $\phi$ satisfying $\phi(0) \leq 2 \phi(1)$.

3. Obviously, if $g$ is a continuous, distribution preserving mapping then $g \circ \ldots \circ g$ is also continuous and distribution preserving. Consequently, any finite iteration of the above $f$ preserves normality.

4. In the $n$-variate case define $\mathbf{f}: \mathbb{R}^{n} \rightarrow \mathbb{R}^{n}$ by $\mathbf{f}(\mathbf{x})=\left(f\left(x_{1}\right), \ldots, f\left(x_{n}\right)\right)$, $\mathbf{x}=\left(x_{1}, \ldots, x_{n}\right) \in \mathbb{R}^{n}$, which is a continuous nonlinear function. If $\mathbf{X}$ is an $n$-variate random vector with iid standard normal components then, obviously, $\mathbf{f}(\mathbf{X}) \stackrel{d}{=} \mathbf{X}$.

Acknowledgements. Thanks are due to D. Gạtarek, who communicated the question, and to R. Wieczorkowski, who helped with Fig. 1.

\section{References}

B. C. Arnold (1979), Some characterizations of the Cauchy distribution, Austral. J. Statist. 21, 166-169. 
N. L. Johnson, S. Kotz and N. Balakrishnan (1994), Continuous Univariate Distributions, Vol. I, Wiley, New York.

I. I. Kotlarski (1967), Problem 6164, Amer. Math. Monthly 84, 575.

M. F. Neuts (1979), Cauchy random variables, (Solution to problem 6164), ibid. 86, 229.

E. J. G. Pitman and E. J. Williams (1967), Cauchy-distributed functions of Cauchy variates, Ann. Math. Statist. 38, 916-918.

L. Shepp (1964), Normal functions of normal random variables, SIAM Rev. 6, 459-460.

E. J. Williams (1969), Cauchy-distributed functions and a characterization of the Cauchy distribution, Ann. Math. Statist. 40, 1083-1085.

Jacek Wesołowski

Mathematical Institute

Warsaw University of Technology

Plac Politechniki 1

00-661 Warszawa, Poland

E-mail: wesolo@alpha.im.pw.edu.pl

Received on 23.5.1996 